OPEN ACCESS

Edited by:

Buck S. Samuel,

Baylor College of Medicine,

United States

Reviewed by:

Levi Morran,

Emory University, United States Ransome Vanzil Van Der Hoeven, University of Texas Health Science Center at Houston, United States

${ }^{*}$ Correspondence: Anke Kloock

anke.kloock@gmail.com

${ }^{\dagger}$ Present address:

Anke Kloock,

Skirball Institute of Biomolecular Medicine, Departments of Cell Biology and Pathology, New York University School of Medicine, New York, NY, United States Lena Peters,

Department of Evolutionary Ecology and Genetics, Christian-AlbrechtsUniversität zu Kiel, Kiel, Germany

Charlotte Elizabeth Rafaluk-Mohr, Institute of Biology, Freie Universität, Berlin, Germany

Specialty section:

This article was submitted to Microbiome in Health and Disease,

a section of the journal

Frontiers in Cellular

and Infection Microbiology

Received: 22 May 2021 Accepted: 10 September 2021 Published: 07 October 2021

Citation:

Kloock A, Peters $L$ and Rafaluk-Mohr C (2021) Sex Matters:

Effects of Sex and Mating in the Presence and Absence

of a Protective Microbe.

Front. Cell. Infect. Microbiol. 11:713387.

doi: 10.3389/fcimb.2021.713387

\section{Sex Matters: Effects of Sex and Mating in the Presence and Absence of a Protective Microbe}

\author{
Anke Kloock ${ }^{* \dagger}$, Lena Peters ${ }^{\dagger}$ and Charlotte Rafaluk-Mohr ${ }^{\dagger}$ \\ Department of Zoology, University of Oxford, Oxford, United Kingdom
}

In most animals, female investment in offspring production is greater than for males. Lifetime reproductive success (LRS) is predicted to be optimized in females through extended lifespans to maximize reproductive events by increased investment in immunity. Males, however, maximize lifetime reproductive success by obtaining as many matings as possible. In populations consisting of mainly hermaphrodites, optimization of reproductive success may be primarily influenced by gamete and resource availability. Microbemediated protection (MMP) is known to affect both immunity and reproduction, but whether sex influences the response to MMP remains to be explored. Here, we investigated the sex-specific differences in survival, behavior, and timing of offspring production between feminized hermaphrodite (female) and male Caenorhabditis elegans following pathogenic infection with Staphylococcus aureus with or without MMP by Enterococcus faecalis. Overall, female survival decreased with increased mating. With MMP, females increased investment into offspring production, while males displayed higher behavioral activity. MMP was furthermore able to dampen costs that females experience due to mating with males. These results demonstrate that strategies employed under pathogen infection with and without MMP are sex dependent.

Keywords: defensive mutualism, sexual immune dimorphism, microbe-mediated protection, host-pathogen interaction, heterogeneity, protection

\section{INTRODUCTION}

In dioecious organisms, females and males invest energy differently throughout their life: In most sexually reproducing species, females and males pay different costs for mating (Rolff, 2002; Rolff et al., 2005). While males invest more energy into mating [by displaying mate searching behavior (Lipton et al., 2004) or outcompeting other males and their sperm (LaMunyon and Ward, 1995)], females generally invest more energy into producing high-quality offspring (Bateman, 1948; Trivers, 1972; Clutton-Brock, 1988). Females try to maximize their reproductive lifespan, while males maximize their sperm output, which is described as Bateman's principle (Bateman, 1948; Trivers, 1972; Clutton-Brock, 1988). Due to these differences in energy investment into mating, the costs and benefits of investment into immunity differ between sexes (Sheldon and Verhulst, 1996; McKean and Nunney, 2001; Lindsey and Altizer, 2009). While males of different species have a reduced survival and body size upon infection with pathogens (such as Panorpa vulgaris, Caenorhabditis elegans, and Daphnia magna after infection with Micrococcus luteus, Bacillus thuringiensis, and 
Octosporea bayeri, respectively), females tend to invest more heavily in immunity (Kurtz et al., 2000; Roth et al., 2008; Zuk, 2009; Masri et al., 2013). Female scorpion flies have increased immune activity (Kurtz et al., 2000), and women survive pandemics (Zuk, 2009), slavery, or famines better than men do (Zarulli et al., 2018). Males, on the other hand, tend to invest more heavily in behavioral traits such as mate-search behavior (Hemptinne et al., 1996; Lipton et al., 2004) and pathogen avoidance (Masri et al., 2013).

In species where the majority of individuals are self-fertilizing hermaphrodites, the situation is more complex as mating is not necessary to maximize fecundity (Brenner, 1974), although similarly to females (Bateman, 1948), fecundity is likely to be primarily dependent on energy availability if gametes are not limited. In self-fertilizing hermaphrodites, such as C. elegans, for whom mating is not necessary for reproduction but males remain in the population, hermaphrodites may be even more "coy" and "choosy" than females in dioecious populations, potentially causing more extreme differences in investment strategies following pathogen infection.

We thus hypothesized that the benefit of a protective microbe would differ among sexes. Protective microbes can be important in host defense in the face of infection, a phenomenon referred to as "defensive mutualism" (King, 2019), where microbes can supplement the host's immune system (Abt and Artis, 2013). Defensive mutualisms have been observed across kingdoms [reviewed in (Ford and King, 2016)]. The potential of defensive mutualism to enhance survival as well as offspring production has been observed repeatedly (Koehler et al., 2013; King et al., 2016; Kloock et al., 2020). However, most of these examples have only considered population-level effects, while few studies have focused on individual behaviors and/or sex differences between the hosts (McLean et al., 2018).

To test for differences in survival, behavior, and timing of offspring production between the two host sexes with or without microbe-mediated protection (MMP) during infection, we used an established experimental system using Caenorhabditis elegans as a host, Enterococcus faecalis as a protective microbe, and pathogenic Staphylococcus aureus (Ford et al., 2016; King et al., 2016; Rafaluk-Mohr et al., 2018). Once C. elegans worms are exposed to E. faecalis and S. aureus, E. faecalis provides microbemediated protection (MMP) by scavenging siderophores from S. aureus (Ford et al., 2016). Here, we used a population of C. elegans made up of males and feminized hermaphrodites, which only carry eggs and cannot produce sperm, and thus are referred to as females (Theologidis et al., 2014). To test for the effect of mating on traits such as survival after pathogen infection and over a lifetime, behavior upon different mating and population structures, and the timing of offspring production, we separated females and males and manipulated the time frame that they could come into contact and mate to be either unmated, short-term mated, or lifetime mated. To test the impact of MMP, worms were exposed to one of three bacterial diets: food only, pathogen without MMP, or pathogen with MMP. We investigated the differences in survival, behavior, and timing of offspring production for the two sexes under the different mating and bacterial diet conditions. We predicted that feminized hermaphrodites would benefit more from the provision of MMP, as feminized hermaphrodites experience costs of the provision of eggs and mating. We furthermore predicted that males would benefit differently from the provision of MMP either by differences in survival or mate searching activity. Our predictions were met, when we observed, that females highly benefit from MMP and show increased survival after pathogen infection and differences in offspring timing, while males are benefiting from MMP by displaying higher mate searching behavior. MMP is able to dampen the costs that females pay by being mated by males.

\section{MATERIALS AND METHODS}

\section{Worm and Bacteria System}

We used an obligate outcrossing worm population [line EEVD00 from Henrique Teotonio (Theologidis et al., 2014)] where worms carry the fog-2(q71) mutation, preventing hermaphrodites from producing sperm (Theologidis et al., 2014). Worms were kept on Nematode Growth Medium (NGM) (Brenner, 1974), and fed with nonpathogenic Salmonella enterica, hereafter referred to as food (Diaz et al., 2015; Desai et al., 2019; Kloock et al., 2020). For pathogenic infection, the Gram-positive Staphylococcus aureus strain MSSA476 (Holden et al., 2004) was used. The strain OG1RF of Enterococcus faecalis (Garsin et al., 2001) was used as a protective microbe against $S$. aureus infection (Ford et al., 2016; King et al., 2016; Rafaluk-Mohr et al., 2018). E. faecalis positively affects host survival, even in the absence of the pathogen S. aureus (Kloock et al., 2020).

\section{Pathogenic Infection and Long-Term Survival Analysis}

All assays were carried out blind. All results shown for food and pathogenic infection with or without MMP have been generated from different setups for each bacterial diet. Results thus cannot be compared between different diets but only within.

Worms were sterilized and synchronized via bleaching (Stiernagle, 2006). Simultaneously, the bacteria were grown in overnight cultures: either E. faecalis overnight in $25 \mathrm{ml}$ of ToddHewitt Broth (THB) or food in $25 \mathrm{ml}$ of Lysogeny broth (LB), both at $30^{\circ} \mathrm{C}$ in a shaking incubator. NGM plates $(6 \mathrm{~cm})$ were inoculated with either $400 \mu \mathrm{l}$ of food or $200 \mu \mathrm{l}$ of food mixed with $200 \mu \mathrm{l}$ of E. faecalis. A total of $600 \mathrm{~L} 1$ worms were added to each NGM plate and kept at $20^{\circ} \mathrm{C}$ for $42 \mathrm{~h}$. Simultaneously, a liquid culture of $S$. aureus was grown in THB from a frozen stock, while food was grown in LB. Both cultures were incubated under shaking conditions at $30^{\circ} \mathrm{C}$ overnight. The following day, $20 \mu \mathrm{l}$ of $S$. aureus overnight culture was pipetted onto $3 \mathrm{~cm}$ on Tryptone Soy Broth agar (TSB) plates and incubated at $30^{\circ} \mathrm{C}$ overnight. Simultaneously, $6 \mathrm{~cm}$ NGM plates were inoculated with $150 \mu \mathrm{l}$ food. These plates were used to split worms into groups of only females, only males, or 50:50 mixed for $6-8 \mathrm{~h}$ (time point when 
the first eggs appeared on the plate) (Table S1). After the worms had mated, 50 worms were placed onto the $S$. aureus lawn with a platinum wire pick and left at $25^{\circ} \mathrm{C}$ for $24 \mathrm{~h}$ (Figure S1) in groups of unmated, short-term mated, and lifetime mated individuals of both sexes (Table S1).

Survival upon pathogenic infection was scored after $24 \mathrm{~h}$. Worms were considered dead if they did not respond to touch with a platinum wire pick. After survival was scored, 10 worms were transferred to $3 \mathrm{~cm}$ NGM plates seeded with $150 \mu$ food and placed at $25^{\circ} \mathrm{C}$. Worms were transferred to new plates every $24 \mathrm{~h}$ with a platinum wire until no further offspring production occurred. Survival was scored every day until all worms were dead. For the food-alone treatment, the long-term survival assay followed a similar protocol except that the experimental procedure was carried out at $20^{\circ} \mathrm{C}$, as is standard for C. elegans (Amrit et al., 2014).

\section{Activity Analysis}

Males and females were previously observed to show different activities, may it be due to infection (Masri et al., 2013) or due to higher mate searching behavior (Lipton et al., 2004). After $24 \mathrm{~h}$ on the pathogen infection plates, worm behavioral activity was determined via calculating the fraction of worms at the edge of the plate. Worms were considered at the edge of the plate if they could not be seen from above.

\section{Avoidance Analysis}

Avoidance behavior is known as a response to pathogen defense (Pees et al., 2017). The proportion of missing worms was calculated $24 \mathrm{~h}$ after pathogen infection and at each transfer in the long-term survival analysis, as previously described (Pees et al., 2017). To define the proportion of missing worms, the number of initially exposed worms minus the counted alive and counted dead worms was divided by the number of initially exposed worms. For the long-term avoidance analysis, the cumulative number of dead worms was used, while only the last time point of each experiment was plotted (Figure S2).

\section{Offspring Production}

The presence or absence of viable offspring on a plate was noted during pathogen infection and during long-term survival. Unmated females might sometimes produce and lay unfertilized eggs, which do not develop into viable larvae (Singaravelu and Singson, 2011). Due to feasibility, the exact numbers of viable offspring could not be assessed. Offspring production was defined as a proportion of plates that had viable offspring over the total amount of plates per treatment.

\section{Statistical Analysis}

Statistical analyses were carried out with RStudio (Version 1.1.463 for Mac) (RStudio Team, 2020). Figures were created with the ggplot2 package (Version 2.1.0). All data, except for the long-term survival and offspring production data, were analyzed with generalized linear models to test for an effect of sex, the mating status, or an interaction between the two. If the interaction had a significant effect, a Tukey multiplecomparison tests (R package multcomp) (Hothorn et al., 2008) was performed (Table S1). The long-term survival data were analyzed with Kaplan-Meier log rank test with FDR correction for multiple testing (Therneau and Grambsch, 2002; Therneau, 2020). The offspring data were analyzed using a Wilcoxon rank test (Mann and Whitney, 1947).

\section{RESULTS AND DISCUSSION}

\section{Lifetime Mated Females Are Most Affected by Pathogen Infection and Mating}

During pathogen infection without (Figure 1A) and with MMP (Figure 1B), males survived better than females (GLM, $X^{2}=$ 172.383, $\mathrm{df}=1, \mathrm{p}<0.001$, and GLM, $\mathrm{X}^{2}=34.383, \mathrm{df}=1, \mathrm{p}<0.001$, respectively), while over a lifetime, this pattern was only present without MMP (Figures 1C-E; without MMP: $\mathrm{p}<0.001$, KaplanMeier survival estimates (KMSE); on food: $\mathrm{p}=0.62$ (KMSE), with MMP: $p=0.32$, (KMSE)). Females were dramatically affected by the mating status, during pathogen infection without and with MMP ( $\mathrm{p}<0.001, \mathrm{GLM}, \mathrm{X}^{2}=108.757, \mathrm{df}=2, \mathrm{p}<0.001$ (Figure 1A), GLM, $\mathrm{X}^{2}=27.152, \mathrm{df}=2, \mathrm{p}<0.001$ (Figure 1B), respectively) and also over a lifetime independent of the bacterial diet (Figures 1C-E), where lifetime mated females survived worse than their unmated or short-term mated counterparts (on food, without MMP and with MMP both comparisons $\mathrm{p}<0.001)$. Males were not affected by mating neither during pathogen infection nor over a lifetime (all $\mathrm{p}>0.05$ ).

Females suffer worse the longer they have been mated with males, while males survive pathogen infection better than females independent of MMP (Figures 1A, B). This pattern holds during pathogen infection (Figures 1A, B) and over a lifetime (Figures 1C-E). The extreme differences we see here may be partially explained by the fact that the females used in our experiments evolved at least in their recent evolutionary history as hermaphrodites that would only occasionally outcross with males and thus are adapted to a far lower frequency of mating (Theologidis et al., 2014). The potential of MMP to enhance survival (King et al., 2016; Martinez et al., 2016; Kloock et al., 2020) as well as offspring production (Koehler et al., 2013) has been shown repeatedly. We expand on this work by investigating whether MMP can mitigate the costs that individuals pay for mating with males. So far, these effects have mainly been considered at the population level. However, the role of MMP might have different effects on individual behaviors of different sexes in different life stages (McLean et al., 2018).

A potential explanation for the observed phenotype could be mechanical gut integrity, which can be different between males and females as observed in Drosophila (Regan et al., 2016). The pathogen used here, $S$. aureus, is known to accumulate in the worms' gut and to kill worms by distention of the intestinal 

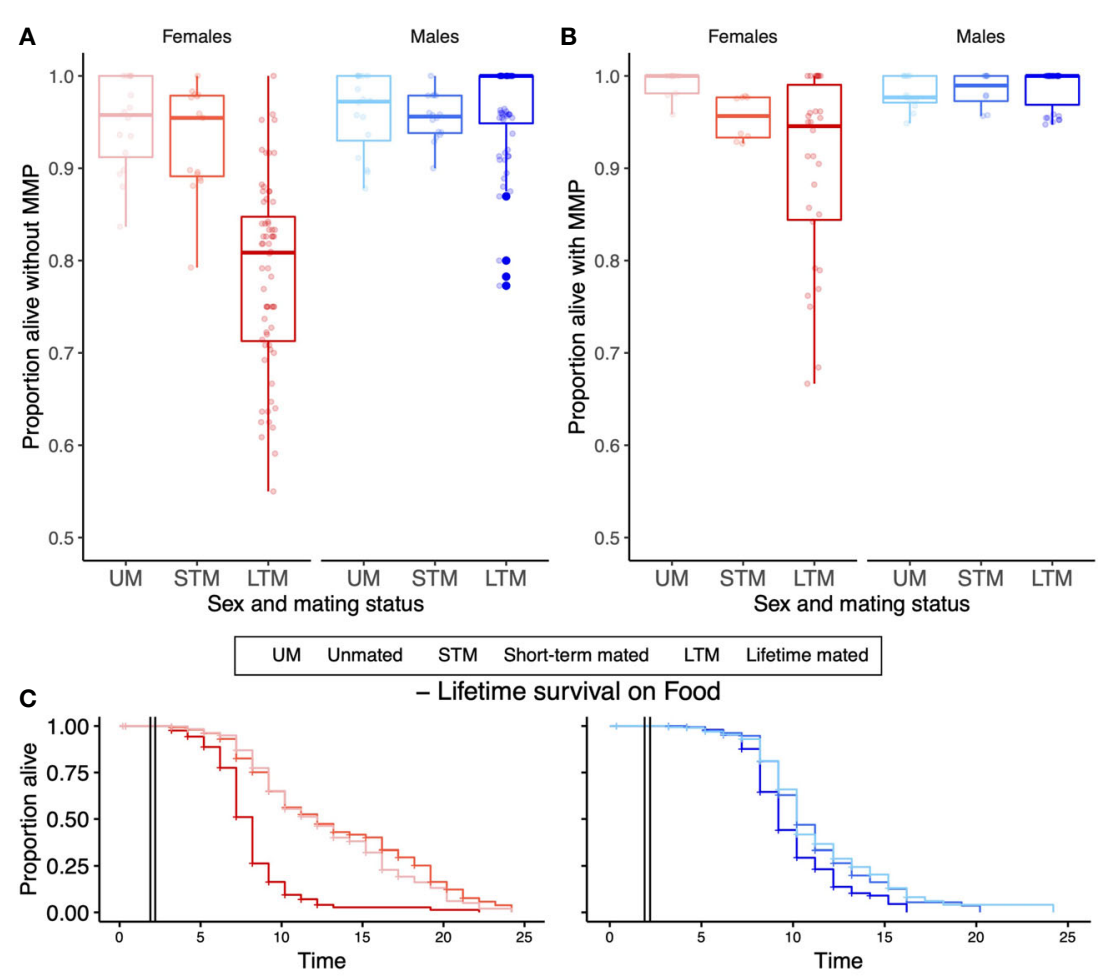

D

- Lifetime survival without MMP
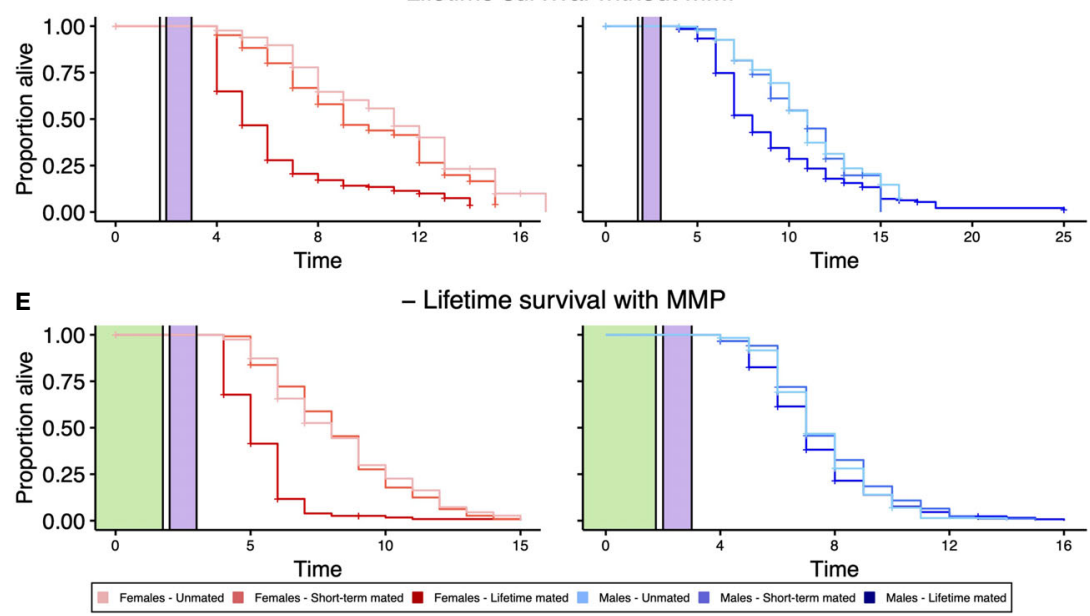

FIGURE 1 | Survival of different sexes and mating treatments after 24-h pathogen infection (A, B) and lifelong (C-E). (A) Without MMP, females suffer more from mating than males do, while males survive better overall. (B) With MMP, females are suffering more from mating, while males survive better overall. (C) When only ever being exposed to food (in white), lifetime mated females survive worse than any other females, while only lifetime mated males survive worse than short-term mated males. (D) After pathogen infection (in purple) without MMP, lifetime mated females survive worse than short-term mated females, which survive worse than unmated females. Male survival was not affected by mating, while males live overall longer than females do. (E) After pathogen infection with MMP (in green), lifetime mated females survive worse than short-term mated females, while males are not affected by mating. (A, B) Boxplots display four biological replicates and three or four technical replicates with 50 worms on each plate. (C-E) Each curve represents the Kaplan-Meier survival estimate for three or four technical replicates and four or five biological replicates with 20 (C) or 10 worms (D, E) on each plate. Vertical lines indicate when worms were transferred to a new bacterial diet.

lumen (Sifri et al., 2003). If gut integrity would thus be more easily damaged in C. elegans females, but not in males (personal observation, Figure S3), this could serve as a potential explanation as to why females are more harshly affected by pathogenic infection with $S$. aureus. The potentially disrupted gut integrity by $S$. aureus infection could further be weakened by mechanical penetration by males, which can shorten hermaphrodites' lifespan (Gems and Riddle, 1996). Whether 
this hypothesis holds true remains to be tested. Although it is possible that due to different gut structures the absolute numbers of protective and pathogenic microbes might differ, we do know that both sexes benefit from protection and due to proliferation in the gut and colonization levels in both sexes are high (Figure S5). Although females carry significantly higher protective microbe loads than males $(\mathrm{LM}$, Sum $\mathrm{Sq}=24.552, \mathrm{Df}=1$, F-value $=17.6003, \mathrm{p}<0.001$ ), females are $30 \%$ larger than males (Riddle et al., 1997), which is proportional to the differences seen in bacterial loads (Figure S5). We thus would expect levels of protection to be similar.

In the absence of hermaphrodites, C. elegans males show highly reduced lifespan (Gems and Riddle, 1996; Shi et al., 2017). The presence of only the male pheromones was sufficient to shorten hermaphrodite (Maures et al., 2014) and male lifespan (Shi et al., 2017). We can, however, only observe this effect for the food-alone treatment (Figure 1C) when lifetime mated males survive significantly longer than unmated or short-term mated males (Figure 1C and Table S2). This life-shortening effect of single-housed males could not be observed after pathogen infection with or without MMP (Figures 1D, E). Both the infection with $S$. aureus and mating reduce the amount of lipids in the worm (Shi et al., 2017; Dasgupta et al., 2020). Possibly the lack of male lifespan reduction in single sex plates after pathogen infection could be linked to this lipid reduction, even though further studies are needed to determine this effect.

The act of mating is costly and life shorting (Gems and Riddle, 1996), independent of offspring production, as here short-term mated females that also produce costly offspring do not have lower survival than unmated females over a lifetime (Figures 1C-E) (Maures et al., 2014; Shi and Murphy, 2014). In C. elegans, the lifeshortening effect is not solely due to offspring production, as even without physical contact or successful mating, hermaphrodites showed reduced lifespan (Maures et al., 2014). During mating, males transfer seminal fluids alongside sperm, which reduces female life in C. elegans and other species and can leave females immune-suppressed post mating (Rolff and Siva-Jothy, 2003). Furthermore, the presence of MMP is able to dampen the costs that females have to pay by being mated with males. Our results reflect a wealth of findings in other species that has shown mating to be costly, such as in Drosophila (Fowler and Partridge, 1989) or birds (Liker and Székely, 2005).

\section{Male and Female Behavior Is Linked to the Presence of the Other Sex}

Females and males respond differently to infection and also display different behavior in the face of infection. Without MMP, those plates with both sexes display higher activity than those plates with unmated or short-term mated individuals (GLM, $\mathrm{X}^{2}=$ 197.14, $d f=5, p<0.001$, Figure 2B), while with MMP, unmated individuals showed significantly decreased activity in comparison to unmated or short-term mated individuals (GLM, $\mathrm{X}^{2}=47.812 .14, \mathrm{df}=2, \mathrm{p}<0.001$, Figure 2C). As this increased activity for lifetime mating worms can be a hint to increased avoidance behavior, which is itself a mechanism to respond to pathogenic infection (Pees et al., 2017), we also assessed the proportion of missing worms during pathogen infection (Figures 2D, E) and over a lifetime (Figures 2F-H). Males went missing with a higher proportion than females during pathogen infection independent of MMP (without MMP: GLM, $\mathrm{X}^{2}=6.4367, \mathrm{df}=1, \mathrm{p}=0.04002$ and with MMP: GLM, $\left.\mathrm{X}^{2}=37.903, \mathrm{df}=1, \mathrm{p}<0.001\right)$ and over a lifetime on food alone (GLM, $\left.\mathrm{X}^{2}=195.959, \mathrm{df}=1, \mathrm{p}<0.001\right)$ and with MMP (GLM, $\mathrm{X}^{2}=160.466, \mathrm{df}=1, \mathrm{p}=0.002$ ), while the difference between the sexes was not significant after pathogen infection without MMP (GLM, $\mathrm{X}^{2}=2.6875, \mathrm{df}=1, \mathrm{p}=0.10$ ).

The activity level was mainly determined by the mating status, while the proportion of missing worms was predominantly determined by sex (Figure 2). Infected females might not be able to move around as much as healthy females, as only healthy females and males on food alone show effects of mating (Figure 2). Furthermore, due to their evolutionary history as hermaphrodites, females may have a decreased drive to look for mates, as mate searching would be largely unnecessary in wildtype populations of self-fertilizing hermaphrodites. The mating status also affected the proportion of missing worms in opposite directions in lifetime mated worms on food: females would be missing with a higher proportion with males present while males would stay with a higher proportion with females present. A parsimonious explanation is that males exhibit higher mate searching behavior, and this is increased when females are not present (Lipton et al., 2004). The proportion of missing worms observed here does not appear to be a consequence of pathogen avoidance behavior (Pees et al., 2017), as this behavior is not observed for pathogen infection without MMP but might instead be dependent on the population structure on the plate.

\section{MMP Enables Females to Invest in Offspring Production During Pathogenic Infection}

Pre-mated females and non-pre-mated females did not start to produce offspring at the same time (Figure 3). Without MMP, pre-mated females did not start to produce offspring during pathogen infection, while pre-mated females did (Wilcoxon rank test (WRT), $W=57.5, p=0.004$, Figure 3A). In the presence of MMP, all females start producing offspring at the same time (WRT, $W=18, p=1$, Figure 3B), independent of their pre-mating status. This pattern could indicate that if females have not yet been mated prior to pathogen infection without MMP, they do not pursue offspring production during infection. They might potentially put reproduction on hold during less favorable conditions and resume reproduction once conditions are more favorable. This can be observed, when over a lifetime, pre-mated and not pre-mated females do not differ in the length of offspring production on each bacterial diet (Figure S4). This is in contrast to previous findings that show fecundity compensation in response to $S$. aureus infection in C. elegans (Pike et al., 2019). This is likely because we only scored for the presence/absence of offspring at a set time point and thus would not be able to detect any change of timing or amount in offspring production, as was described for fecundity compensation in this system of C. elegans and S. aureus (Pike et al., 2019). 


\section{Proportion of worms at the edge}

A

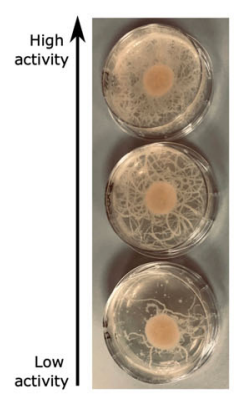

B

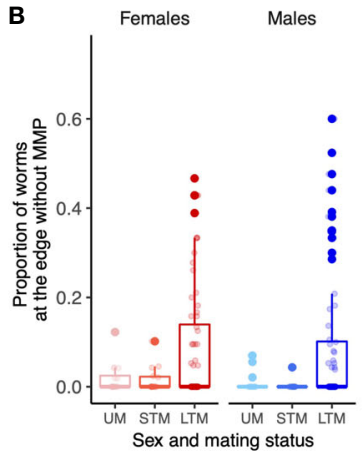

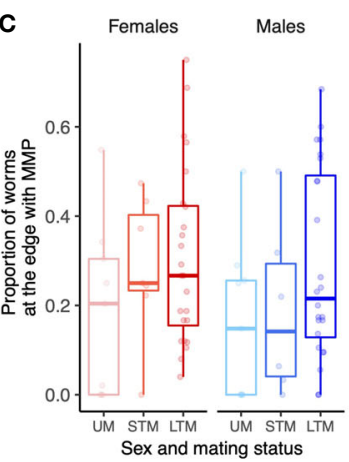

Proportion of missing worms after pathogen infection
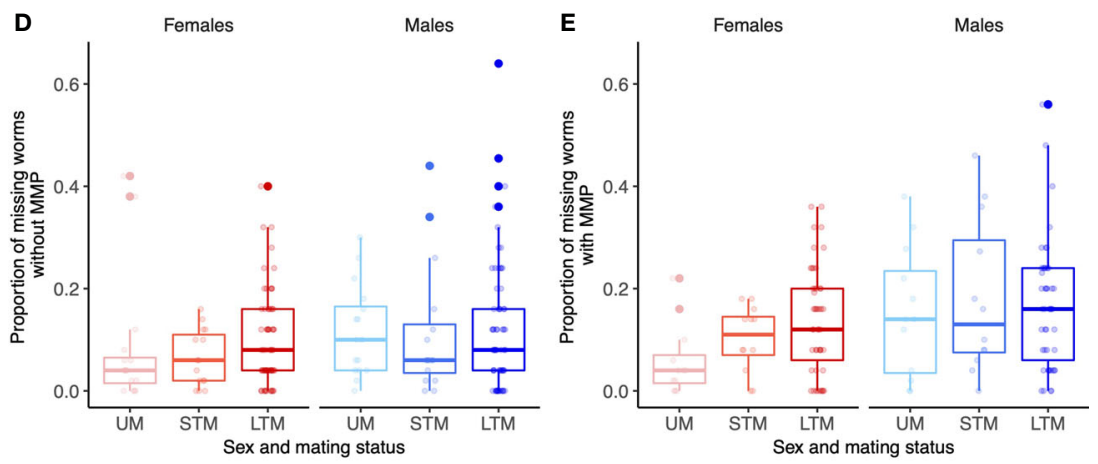

Proportion of missing worms after lifetime

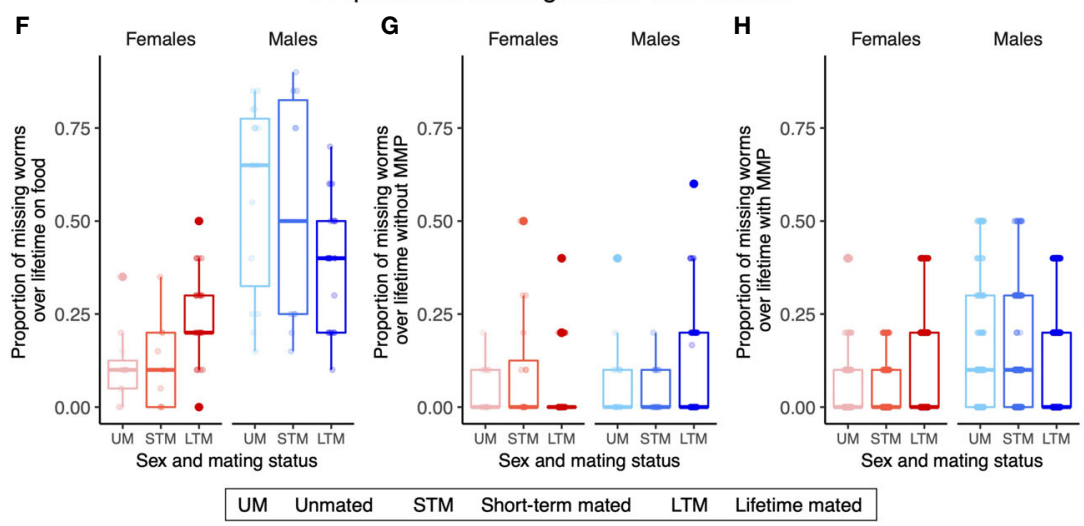

FIGURE 2 | Female and male behavior is linked to the presence of the other sex. (A) Picture of three pathogenic plates with different activity levels from low to high. (B) Without MMP after pathogen infection, plates with lifetime mated worms of both sexes had a higher proportion of worms at the edge of the plate. (C) With MMP after pathogen infection, plates with lifetime mated worms of both sexes have a higher proportion of worms at the edge of the plate. Proportion of missing worms of different sexes and mating treatments either during pathogen infection (D, E) and over lifetime (F-H, only the last time point is plotted). (D) During pathogen infection without MMP, a higher proportion of males are missing than of females. (E) During pathogen infection with MMP, more males went missing than females. (F) Proportion of missing worms on food over lifetime. Females showed lower proportion of missing worms than males. (G) Proportion of missing worms without MMP over lifetime with no differences detected. (H) Proportion of missing worms with MMP over lifetime, where males have a higher proportion of missing worms than females do. (B-H) Boxplots represent the mean \pm the standard error of the mean of four or five biological replicates and three or four technical replicates with 50 (B-E), 20 (F), or 10 worms $(\mathbf{G}, \mathbf{H})$ on each plate. $(\mathbf{F}-\mathbf{H})$ Changes in the proportion of missing worms over time can be found in Figure $\mathbf{S 2}$.

\section{CONCLUSION}

In conclusion, female survival decreases with increasing mating with males, while male survival was unaffected by mating after pathogen infection. The two sexes benefit from MMP differently.
With MMP, females invest more energy into egg production, while males invest more into mate searching behavior. These results are likely to be enhanced in the results we see here as females were artificially feminized, having evolved as hermaphrodites. Furthermore, MMP is able to dampen the 


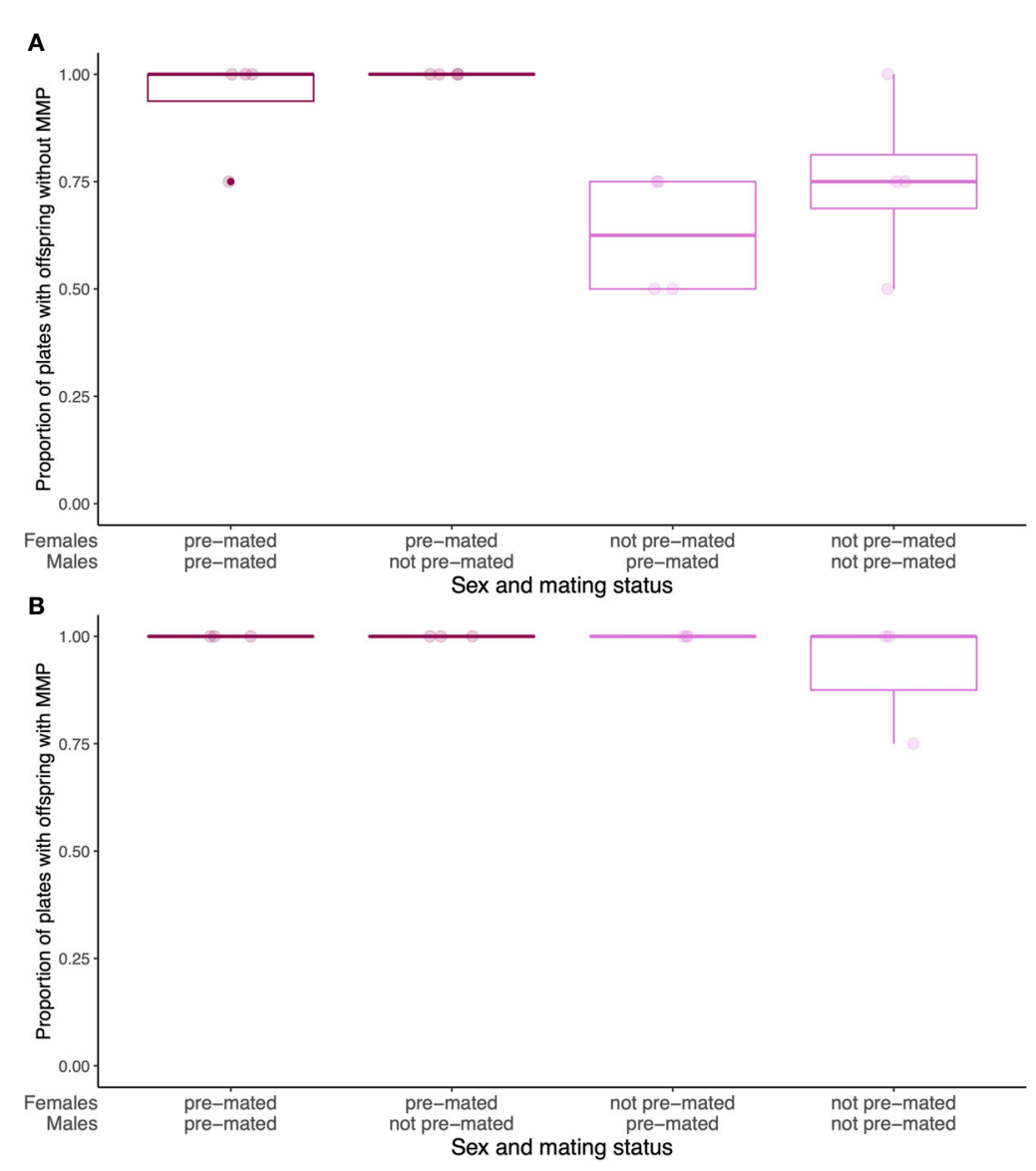

FIGURE 3 | Differences in timing of offspring production during pathogen infection without (A) and with (B) MMP (A). During pathogen infection but without MMP, there are fewer plates with offspring, despite the presence of males. (B) During pathogen infection, but with MMP, all plates with pre-mated and not pre-mated females are producing offspring. Boxplots represent three or four technical replicates with 25 females and 25 males on each plate.

costs that females pay by being mated by males. This study highlights that in spite of consistent population level responses to defensive mutualists, individual variation depends heavily on diet, sex, mating status, and interaction of these factors. Even though defensive mutualists provide benefits to the host, for females, mating comes at a high cost.

\section{DATA AVAILABILITY STATEMENT}

The datasets presented in this study can be found in online repositories. The names of the repository/repositories and accession number(s) can be found below: https://osf.io/bnwrt/, Open Science Framework.

\section{AUTHOR CONTRIBUTIONS}

$\mathrm{AK}$ and CR-M conceived and designed the project. AK and LP performed all experiments. AK and CR-M performed the statistical analysis. AK and CR-M wrote the manuscript and all authors agreed on the final version. All authors contributed to the article and approved the submitted version.

\section{FUNDING}

AK was supported by a fellowship from the "Studienstiftung des Deutschen Volkes". LP was supported by an ERASMUS + fellowship. This work was funded by a Leverhulme Trust project grant (RPG-2015-165) to Kayla King.

\section{ACKNOWLEDGMENTS}

We would like to thank Kayla King for providing funding, laboratory space, and intellectual support for the project and the King group for support throughout the laboratory experiments, particularly Maria Ordovas-Montanes and Andrea Gomez Charmorro. We would also like to thank Michael Bonsall for valuable discussion and feedback on the analysis. We are also grateful for reviewer comments. 


\section{SUPPLEMENTARY MATERIAL}

The Supplementary Material for this article can be found online at: https://www.frontiersin.org/articles/10.3389/fcimb.2021. 713387/full\#supplementary-material

Supplementary Figure 1 | Experimental procedure. Starved worms were transferred to new NGM plates seeded with food 2.5 days before bleaching to allow for egg laying. Worms were then bleached as described previously (Stiernagle, 2006) and left in M9 buffer overnight to allow for larvae to hatch. Simultaneously, the bacteria on which worms were raised to $L 4$ stage were grown in overnight cultures: Either E. faecalis overnight in $25 \mathrm{ml}$ of Todd-Hewitt Broth (THB), or food in $25 \mathrm{ml}$ of Lysogeny broth (LB), both at $30{ }^{\circ} \mathrm{C}$ in a shaking incubator. Subsequently, $6 \mathrm{~cm}$

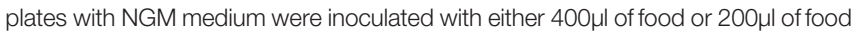
mixed with $200 \mu \mathrm{l}$ of $E$. faecalis. Plates with freshly inoculated bacteria were dried at room temperature before approximately $600 \mathrm{~L} 1$ worms were added to each NGM plate and transferred to $20^{\circ} \mathrm{C}$ for $42 \mathrm{~h}$. At the same time, a liquid culture of $S$. aureus was grown in THB from frozen stock, while food was grown in LB. Both cultures were incubated under shaking conditions at $30^{\circ} \mathrm{C}$ overnight. The following day, $20 \mu \mathrm{l}$ of the $S$. aureus overnight culture was pipetted onto $3 \mathrm{~cm}$ on Tryptone Soy Broth agar (TSB) plates. Simultaneously, $6 \mathrm{~cm}$ NGM plates were inoculated with 150 $\mathrm{\mu l}$ food. These plates were used to split worms into groups of only females, only males or 50:50 mixed for 6-8h (time point when the first eggs appeared on the plate) as outlined in Table S1. For S. aureus infection, 50 worms were picked on pathogen exposure plates and left at $25^{\circ} \mathrm{C}$ for $24 \mathrm{~h}$. Survival was scored after $24 \mathrm{~h}$ with all alive and dead worms present on the plates counted. Worms were considered dead if they do not respond to touch with a platinum wire pick. After survival was scored, 10 worms were transferred to $3 \mathrm{~cm}$ NGM plates seeded with $150 \mu \mathrm{l}$ food and placed at $25^{\circ} \mathrm{C}$. Worms were then picked to new plates every $24 \mathrm{~h}$ until no offspring was produced anymore, to allow for more accurate tracking of individuals. Survival was scored every day until all worms were dead. The lifelong survival assay on food alone was performed as described above, with a few adjustments. The experimental procedure was carried out at $20^{\circ} \mathrm{C}$, and worms were split only females, only males or 50:50 mixed when worms were 46h old. The mixed treatment only consisted of "Females only with Males only" and "50:50 Females with 50:50 Males". Once eggs occurred on the plates, worms were transferred to $6 \mathrm{~cm}$ NGM plates inoculated with $150 \mu \mathrm{l}$ of food.

Supplementary Figure 2 | Livelong proportion of missing worms on food (A), with microbe-mediated protection (B) and without of microbe-mediated protection

\section{REFERENCES}

Abt, M. C., and Artis, D. (2013). The Dynamic Influence of Commensal Bacteria on the Immune Response to Pathogens. Curr. Opin. Microbiol. 16 (1), 4-9. doi: 10.1016/j.mib.2012.12.002

Amrit, F. R. G., Ratnappan, R., Keith, S. A., and Ghazi, A. (2014). The C. Elegans Lifespan Assay Toolkit. Methods 68 (3), 465-475. doi: 10.1016/ j.ymeth.2014.04.002

Bateman, A. J. (1948). Intra-Sexual Selection in Drosophila. Heredity 2, 349-368. doi: 10.1038/hdy.1948.21

Brenner, S. (1974). The Genetics of Caenorhabditis elegans. Genetics 77 (1), 71-94. doi: $10.1093 /$ genetics/77.1.71

Clutton-Brock, T. H. (1988). Reproductive Success (Chicago: University of Chicago Press).

Dasgupta, M., Shashikanth, M., Bojanala, N., Gupta, A., Javed, S., and Singh, V. (2020). Nuclear Hormone Receptor NHR-49 Shapes Immuno-Metabolic Response of Caenorhabditis Elegans to Enterococcus Faecalis Infection. Infect. Immun. 88, e00130-20. doi: 10.1128/IAI.00130-20

Desai, S. K., Padmanabhan, A., Harshe, S., Zaidel-Bar, R., and Kenney, L. J. (2019). Salmonella Biofilms Program Innate Immunity for Persistence in Caenorhabditis Elegans. Proc. Natl. Acad. Sci. 116 (25), 12462-12467. doi: 10.1073/pnas.1822018116

Diaz, S. A., Mooring, E. Q., Rens, E. G., and Restif, O. (2015). Association With Pathogenic Bacteria Affects Life-History Traits and Population Growth in Caenorhabditis Elegans. Ecol. Evol. 5 (8), 1653-1663. doi: 10.1002/ece3.1461
(C). (A) Females showed lower proportion of missing worms than males on food alone (in white). (B) Proportion of missing worms after pathogen infection (in purple) without MMP over lifetime with no differences detected. (C) Proportion of missing worms with MMP (in green) over lifetime, where males have a higher proportion of missing worms than females do. (A-C) Each point represents the mean \pm the standard error of the mean of four or five biological replicates and three or four technical replicates with $20(\mathrm{~A})$ or 10 worms (B, C) on each plate.

Supplementary Figure 3 | Females show hints of lost gut integrity, while male gut integrity is still intact. Worms that were fed food, coloured with a blue food dye (following a previously described protocol (Gelino et al., 2016). If gut integrity is still fully intact, blue dye can only be seen in the intestine (as in the lower worm - a male), while if the gut integrity is out of balance, the blue dye can be found in the whole worm body cavity (as in the upper worm - a female).

Supplementary Figure 4 | Over a lifetime no difference between pre-mated and not pre-mated females for the days of offspring production can be observed independent on whether worms were raised on food (in orange), infected with the pathogen in the absence (in purple) or presence (in green) of MMP. (A, B) Each point represents the mean \pm the standard error of the mean of three or four technical replicates.

Supplementary Figure 5 | With MMP, females are colonized by more E. faecalis than their male counter parts. After $24 \mathrm{~h}$ infection with $\mathrm{S}$. aureus, worms were filter tip washed as described before (Kloock et al., 2020) to remove all externally attached bacteria. Consequently, worms were crushed open, and the gut content was plated on $E$. faecalis selective medium (TSA + rifampicin), to only count $E$. faecalis colonies. The difference between the two sexes is significant with a linear model (LM) $(\mathrm{LM}, \mathrm{Sum} \mathrm{Sq}=$ 24.552, $\mathrm{Df}=1$, F-value $\left.=17.6003, \mathrm{p}=4.99 \times 10^{-5}\right)$, while neither the mating status nor an interaction of the two show significant differences (both $\mathrm{p}>0.05$ ).

Supplementary Table 1 | Mating Treatments: Three different mating treatments (Unmated, Short-term mated and Lifetime mated) were set up for both sexes (Female in Red and Male in Blue), which were either single sex plates for each sex (Females only or Males only) or a 50:50 mixed population. Worms were left on these mating plates for 6-8h, before three different mating treatments were set up (unmated, short-term mated, and lifetime mated, the darker the colour the longer the mating period) for each sex.

Supplementary Table 2 | Summary of all statistical results. The following abbreviations were used: U, unmated; STM, short-term mated; LTM, Lifetime mated.

Ford, S. A., Kao, D., Williams, D., and King, K. C. (2016). Microbe-Mediated Host Defence Drives the Evolution of Reduced Pathogen Virulence. Nat. Commun. 7 (1), 13430. doi: 10.1038/ncomms 13430

Ford, S. A., and King, K. C. (2016). Harnessing the Power of Defensive Microbes: Evolutionary Implications in Nature and Disease Control. PloS Pathog. 12 (4), e1005465. doi: 10.1371/journal.ppat.1005465

Fowler, K., and Partridge, L. (1989). A Cost of Mating in Female Fruitflies. Nature 338 (6218), 760-761. doi: 10.1038/338760a0

Garsin, D. A., Sifri, C. D., Mylonakis, E., Qin, X., Singh, K. V., Murray, B. E., et al (2001). A Simple Model Host for Identifying Gram-Positive Virulence Factors. PNAS 98, 10892-10897. doi: 10.1073/pnas.191378698

Gelino, S., Chang, J. T., Kumsta, C., She, X., Davis, A., Nguyen, C., et al (2016). Intestinal Autophagy Improves Healthspan and Longevity in C. Elegans During Dietary Restriction. PloS Genet. 12 (7), e1006135. doi: 10.1371/ journal.pgen.1006135

Gems, D., and Riddle, D. L. (1996). Longevity in Caenorhabditis Elegans Reduced by Mating But Not Gamete Production. Nature 379 (6567), 723-725. doi: 10.1038/379723a0

Hemptinne, J.-L., Dixon, A. F. G., and Lognay, G. (1996). Searching Behaviour and Mate Recognition by Males of the Two-Spot Ladybird Beetle, Adalia Bipunctata. Ecol. Entomol. 21 (2), 165-170. doi: 10.1111/j.1365-2311.1996.tb01183.x

Holden, M. T. G., Feil, E. J., Lindsay, J. A., Peacock, S. J., Day, N. P. J., Enright, M. C., et al (2004). Complete Genomes of Two Clinical Staphylococcus Aureus Strains: Evidence for the Rapid Evolution of Virulence and Drug Resistance. Proc. Natl. Acad. Sci. 101 (26), 9786-9791. doi: 10.1073/pnas.0402521101 
Hothorn, T., Bretz, F., and Westfall, P. (2008). Simultaneous Inference in General Parametric Models. Biom. J. 50 (3), 346-363. doi: 10.1002/bimj.200810425

King, K. C. (2019). Defensive Symbionts. Curr. Biol. 29 (3), R78-R80. doi: 10.1016/ j.cub.2018.11.028

King, K. C., Brockhurst, M. A., Vasieva, O., Paterson, S., Betts, A., Ford, S. A., et al (2016). Rapid Evolution of Microbe-Mediated Protection Against Pathogens in a Worm Host. ISME J. 10 (8), 1915-1924. doi: 10.1038/ismej.2015.259

Kloock, A., Bonsall, M. B., and King, K. C. (2020). Evolution and Maintenance of Microbe-Mediated Protection Under Occasional Pathogen Infection. Ecol. Evol. 10, 8634-8642. doi: 10.1002/ece3.6555

Koehler, S., Doubský, J., and Kaltenpoth, M. (2013). Dynamics of SymbiontMediated Antibiotic Production Reveal Efficient Long-Term Protection for Beewolf Offspring. Front. Zool. 10 (1), 3. doi: 10.1186/1742-9994-10-3

Kurtz, J., Wiesner, A., Göth, P., and Sauer, K. P. (2000). Gender Differences and Individual Variation in the Immune System of the Scorpionfly Panorpa Vulgaris (Insecta: Mecoptera). Dev. Comp. Immunol. 12, 1-12. doi: 10.1016/ S0145-305X(99)00057-9

LaMunyon, C. W., and Ward, S. (1995). Sperm Precedence in a Hermaphroditic Nematode (Caenorhabditis Elegans) is Due to Competitive Superiority of Male Sperm. Experientia 51, 817-823. doi: 10.1007/BF01922436

Liker, A., and Székely, T. (2005). Mortality Costs of Sexual Selection and Parental Care in Natural Populations of Birds. Evolution 59 (4), 890-897. doi: 10.1111/ j.0014-3820.2005.tb01762.x

Lindsey, E., and Altizer, S. (2009). Sex Differences in Immune Defenses and Response to Parasitism in Monarch Butterflies. Evol. Ecol. 23 (4), 607-620. doi: $10.1007 / \mathrm{s} 10682-008-9258-0$

Lipton, J., Kleemann, G., Ghosh, R., Lints, R., and Emmons, S. W. (2004). Mate Searching in Caenorhabditis Elegans: A Genetic Model for Sex Drive in a Simple Invertebrate. J. Neurosci. 24 (34), 7427-7434. doi: 10.1523/JNEUROSCI.174604.2004

Mann, H. B., and Whitney, D. R. (1947). On a Test of Whether One of Two Random Variables is Stochastically Larger Than the Other. Ann. Math. Stat. 8 (1), 50-60. doi: 10.1214/aoms/1177730491

Martinez, J., Cogni, R., Cao, C., Smith, S., Illingworth, C. J. R., and Jiggins, F. M. (2016). Addicted? Reduced Host Resistance in Populations With Defensive Symbionts. Proc. R. Soc. B Biol. Sci. 283 (1833), 20160778. doi: 10.1098/rspb.2016.0778

Masri, L., Schulte, R. D., Timmermeyer, N., Thanisch, S., Crummenerl, L. L., Jansen, G., et al (2013). Sex Differences in Host Defence Interfere With Parasite-Mediated Selection for Outcrossing During Host-Parasite Coevolution. Ecol. Lett. 16 (4), 461-468. doi: 10.1111/ele.12068

Maures, T. J., Booth, L. N., Benayoun, B. A., Izrayelit, Y., Schroeder, F. C., and Brunet, A. (2014). Males Shorten the Life Span of C. Elegans Hermaphrodites via Secreted Compounds. Science 343, 541-544. doi: 10.1126/science. 1244160

McKean, K. A., and Nunney, L. (2001). Increased Sexual Activity Reduces Male Immune Function in Drosophila Melanogaster. Proc. Natl. Acad. Sci. 98 (14), 7904-7909. doi: 10.1073/pnas.131216398

McLean, A. H. C., Ferrari, J., and Godfray, H. C. J. (2018). Do Facultative Symbionts Affect Fitness of Pea Aphids in the Sexual Generation? Entomol. Exp. Appl. 166 (1), 32-40. doi: 10.1111/eea.12641

Pees, B., Kloock, A., Nakad, R., Barbosa, C., and Dierking, K. (2017). Enhanced Behavioral Immune Defenses in a C. Elegans C-Type Lectin-Like Domain Gene Mutant. Dev. Comp. Immunol. 74, 237-242. doi: 10.1016/j.dci.2017.04.021

Pike, V. L., Ford, S. A., King, K. C., and Rafaluk-Mohr, C. (2019). Fecundity Compensation is Dependent on the Generalized Stress Response in a Nematode Host. Ecol. Evol. 9 (20), 11957-11961. doi: 10.1002/ece3.5704

Rafaluk-Mohr, C., Ashby, B., Dahan, D. A., and King, K. C. (2018). Mutual Fitness Benefits Arise During Coevolution in a Nematode-Defensive Microbe Model. Evol. Lett. 2 (3), 246-256. doi: 10.1002/evl3.58

Regan, J. C., Khericha, M., Dobson, A. J., Bolukbasi, E., Rattanavirotkul, N., and Partridge, L. (2016). Sex Difference in Pathology of the Ageing Gut Mediates the Greater Response of Female Lifespan to Dietary Restriction. ELife 5, e10956. doi: 10.7554/eLife.10956

Riddle, D. L., Blumenthal, T., Meyer, B. J., and Priess, J. R. (1997). "Sexual Dimporohism," in C. Elegans II, 2. Edition (Cold Spring Harbor (NY)).
Rolff, J. (2002). Bateman's Principle and Immunity. Proc. R. Soc. Lond. B Biol. Sci. 269 (1493), 867-872. doi: 10.1098/rspb.2002.1959

Rolff, J., Armitage, S. A. O., and Coltman, D. W. (2005). Genetic Constraints and Sexual Dimorphism in Immune Defense. Evolution 59 (8), 1844-1850. doi: 10.1111/j.0014-3820.2005.tb01831.x

Rolff, J., and Siva-Jothy, M. T. (2003). Invertebrate Ecological Immunology. Science 301 (5632), 472-475. doi: 10.1126/science.1080623

Roth, O., Ebert, D., Vizoso, D. B., Bieger, A., and Lass, S. (2008). Male-Biased SexRatio Distortion Caused by Octosporea Bayeri, a Vertically and HorizontallyTransmitted Parasite of Daphnia Magna. Int. J. Parasitol. 38 (8-9), 969-979. doi: 10.1016/j.ijpara.2007.11.009

RStudio Team (2020). RStudio. Integrated Development for R (PBC, Boston, MA: RStudio). Available at: http://www.rstudio.com/.

Sheldon, B. C., and Verhulst, S. (1996). Ecological Immunology: Costly Parasite Defences and Trade-Offs in Evolutionary Ecology. Trends Ecol. Evol. 11 (8), 317-321. doi: 10.1016/0169-5347(96)10039-2

Shi, C., and Murphy, C. T. (2014). Mating Induces Shrinking and Death in Caenorhabditis Mothers. Science 343 (6170), 536-540. doi: 10.1126/ science. 1242958

Shi, C., Runnels, A. M., and Murphy, C. T. (2017). Mating and Male Pheromone Kill Caenorhabditis Males Through Distinct Mechanisms. ELife 6, e23493. doi: $10.7554 /$ LLife. 23493

Sifri, C. D., Begun, J., Ausubel, F. M., and Calderwood, S. B. (2003). Caenorhabditis Elegans as a Model Host for Staphylococcus Aureus Pathogenesis. Infect. Immun. 71 (4), 2208-2217. doi: 10.1128/IAI.71.4.2208-2217.2003

Singaravelu, G., and Singson, A. (2011). "New Insights Into the Mechanism of Fertilization in Nematodes," in International Review of Cell and Molecular Biology, vol. 289. (Elsevier), 211-238. doi: 10.1016/B978-0-12-3860392.00006-7

Stiernagle, T. (2006). "Maintenance of C. Elegans," in WormBook. Available at: https://doi.org/10.1895/wormbook.1.101.1.

Theologidis, I., Chelo, I. M., Goy, C., and Teotónio, H. (2014). Reproductive Assurance Drives Transitions to Self-Fertilization in Experimental Caenorhabditis Elegans. BMC Biol. 21 (93), 1-21. doi: 10.1186/s12915-014-0093-1

Therneau, T. M. (2020) A Package for Survival Analysis in R. Available at: https:// CRAN.R-project.org/package $=$ survival.

Therneau, T. M., and Grambsch, P. M. (2002). Modeling Survival Data: Extending the Cox Model Vol. 44 (New York: Springer New York). Available at: http:// www.tandfonline.com/doi/abs/10.1198/tech.2002.s656.

Trivers, R. L. (1972). "Parental Investment and Sexual Selection," in Sexual Selection and the Descent of Man (Chicago: Aldine Publishing Company), 136-175.

Zarulli, V., Barthold Jones, J. A., Oksuzyan, A., Lindahl-Jacobsen, R., Christensen, K., and Vaupel, J. W. (2018). Women Live Longer Than Men Even During Severe Famines and Epidemics. Proc. Natl. Acad. Sci. 115 (4), E832-E840. doi: 10.1073/pnas.1701535115

Zuk, M. (2009). The Sicker Sex. PloS Pathog. 5 (1), e1000267. doi: 10.1371/ journal.ppat.1000267

Conflict of Interest: The authors declare that the research was conducted in the absence of any commercial or financial relationships that could be construed as a potential conflict of interest.

Publisher's Note: All claims expressed in this article are solely those of the authors and do not necessarily represent those of their affiliated organizations, or those of the publisher, the editors and the reviewers. Any product that may be evaluated in this article, or claim that may be made by its manufacturer, is not guaranteed or endorsed by the publisher.

Copyright $\odot 2021$ Kloock, Peters and Rafaluk-Mohr. This is an open-access article distributed under the terms of the Creative Commons Attribution License (CC BY). The use, distribution or reproduction in other forums is permitted, provided the original author(s) and the copyright owner(s) are credited and that the original publication in this journal is cited, in accordance with accepted academic practice. No use, distribution or reproduction is permitted which does not comply with these terms. 